\title{
The NodeHopper: Enabling Low Latency Ranking with Constraints via a Fast Dual Solver
}

\author{
Anton Zhernov* \\ typedef@google.com \\ DeepMind \\ London, UK \\ Dan A. Calian \\ dancalian@google.com \\ DeepMind \\ London, UK
}

\author{
Krishnamurthy (Dj) \\ Dvijotham* \\ dvij@google.com \\ DeepMind \\ London, UK
}

\author{
Michelle Gong \\ michellegong@google.com \\ DeepMind \\ Mountain View, USA
}

Timothy A. Mann

kingtim@google.com

DeepMind

London, UK

\author{
Ivan Lobov* \\ lobov@google.com \\ DeepMind \\ London, UK
}
Natarajan Chandrashekar
natarajanc@google.com Google
Mountain View, USA

\begin{abstract}
Modern recommender systems need to deal with multiple objectives like balancing user engagement with recommending diverse and fresh content. An appealing way to optimally trade these off is by imposing constraints on the ranking according to which items are presented to a user. This results in a constrained ranking optimization problem that can be solved as a linear program (LP). However, off-the-shelf LP solvers are unable to meet the severe latency constraints in systems that serve live traffic. To address this challenge, we exploit the structure of the dual optimization problem to develop a fast solver. We analyze theoretical properties of our solver and show experimentally that it is able to solve constrained ranking problems on synthetic and real-world recommendation datasets an order of magnitude faster than off-the-shelf solvers, thereby enabling their deployment under severe latency constraints.
\end{abstract}

\section{KEYWORDS}

ranking, linear programming, scalability

\section{ACM Reference Format:}

Anton Zhernov, Krishnamurthy (Dj) Dvijotham, Ivan Lobov, Dan A. Calian, Michelle Gong, Natarajan Chandrashekar, and Timothy A. Mann. 2020. The NodeHopper: Enabling Low Latency Ranking with Constraints via a Fast Dual Solver. In Proceedings of the 26th ACM SIGKDD Conference on Knowledge Discovery and Data Mining (KDD '20), August 23-27, 2020, Virtual Event, CA, USA. ACM, New York, NY, USA, 10 pages. https://doi.org/10. $1145 / 3394486.3403181$

*All three authors contributed equally to this research

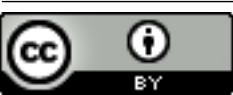

This work is licensed under a Creative Commons Attribution International 4.0 License. KDD '20, August 23-27, 2020, Virtual Event, CA, USA

(c) 2020 Copyright held by the owner/author(s).

ACM ISBN 978-1-4503-7998-4/20/08.

https://doi.org/10.1145/3394486.3403181

\section{INTRODUCTION}

Content ranking is ubiquitous and central to information retrieval [15] and recommender systems [14]. An underlying assumption of many ranking systems is the existence of a single relevance metric. However, in practical applications there are typically multiple aspects of content recommendation that are difficult to capture with a single scalar value [2].

While several approaches have been proposed for learning to rank $[6,21]$, we focus on a different setting - we assume that a scoring function can be learned that predicts the "desirability" of a particular item being considered (for example, its click-through-rate or PageRank score). Given the scoring function we seek to optimize the ranking so as to maximize the expected scoring function (under a probabilistic click model [8]) subject to constraints on the ranking; the constraints could express diversity requirements, fairness requirements or other considerations that are orthogonal to the primary ranking score (for example, freshness of the recommended items in an evolving recommender systems).

These problems can be formulated as a linear program and solved with general purpose solver. However, if we want to incorporate ranking into an application that has a low latency constraint, ranking must be done in less than 100 milliseconds to avoid perceived latency by the user [16]. In web-based applications, the situation is even more severe, since user interactions must also take into consideration the time required to send and receive content for ranking. The implication is that ranking must not take more than 50 milliseconds to be feasible. Unfortunately, general purpose linear programming solvers do not satisfy these strict requirements. In this paper, we introduce a specialized solver for ranking with multiple constraints. With a small number of constraints (up to 10) and hundreds of items to rank, a setting typical for production recommender systems after candidate generation and filtering phases, this specialized solver is an order of magnitude faster than general 
purpose solvers. More importantly, this solver enables using constrained optimization in production by solving these problems well within the range of 50 milliseconds.

\subsection{Contributions}

The main contributions of our paper are summarized as follows: 1. We study the dual formulation of a relaxed constrained ranking We show that by exploiting the structure of the problem we can solve it efficiently.

2. We develop a novel algorithm, the NodeHopper to solve the dual problem efficiently by hopping between "nodes", i.e., points at which the piece-wise linear objective switches from one piece to another. The NodeHopper algorithm can be interpreted as a particular type of constraint generation algorithm [13] tailored for the constrained ranking problem. We establish theoretical convergence guarantees for the NodeHopper.

3. Using experiments on synthetic and real-world datasets, we demonstrate the practical improvements in speed from using our solver vs. state-of-the-art off-the shelf LP solvers. We obtain speed ups of $10-100 \times$ for the ranking problems under consideration. This enables the application of constrained ranking in the latency constrained applications discussed earlier.

\subsection{Related work}

The literature on learning for ranking problems with constraints can be organized into the following categories:

Learning and ranking with specific constraints: There has also been work on ranking with specific classes of constraints like diversity [17] or fairness [4, 7]. However, these approaches do not generalize to arbitrary constraints and rely on specific assumptions about the structure of the constraints.

Ranking with system-wide constraints: Another class of approaches studies the problem of ensuring that, on average, over several requests served by a recommender system, there are certain system-wide constraints that are met [18]. These approaches do not guarantee that constraints are met on a per-request basis, only on average over a large number of requests. This has the disadvantage that different users can have a very different experience: for example, some users may see a much higher fraction of diverse content recommended, while others may repeatedly receive similar content with low diversity.

Ranking with general exposure constraints: The closest work to ours are approaches that take the output of an ML based ranking/scoring function and use an optimization problem to compute a ranking that can be shown to satisfy constraints. These approaches assume a probabilistic model of user attention (like [8]) which model a position dependent attention bias of the user (that decays from left to right, top to bottom etc. given a list of recommendations). Given item-wise values for a certain metric of interest, they are then able to enforce constraints which require that the expected value of the metric (under a specific model of position-dependent attention or exposure probabilities) is larger than a threshold [1, 10,19] - these have been termed exposure constraints in previous work [19]. This has a distinct advantage of being able to guarantee that constraints are satisfied on a per-request basis, i.e. every time the system makes a list of recommendations to a user, the recommendations made are guaranteed to satisfy a set of constraints (albeit probabilistically).

The closest work to our own is that of Singh and Joachims [19]. Our formulation of the constrained ranking problem is nearly identically to theirs, with one additional restriction that the attention model is shared across all the metrics being considered. This restriction is quite natural but allows us to significantly speed up the computation of the optimal constrained ranking solution, achieving an order of magnitude speedup over competing approaches that leverage off-the-shelf optimization algorithms.

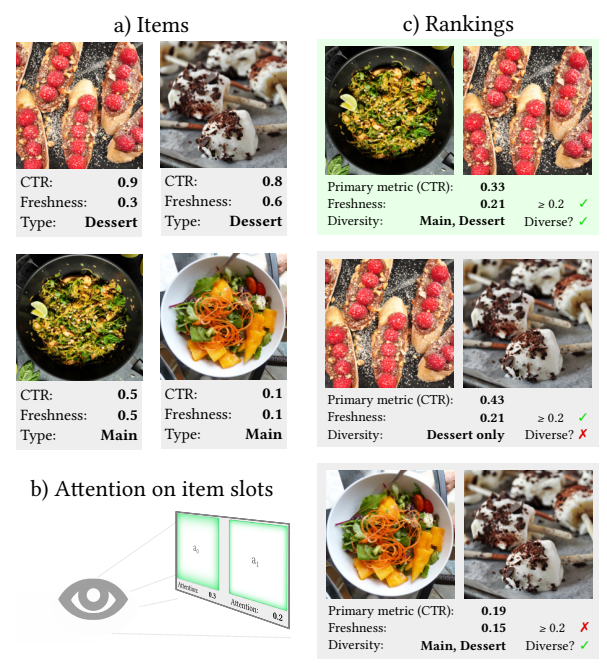

Figure 1: Example of a recommender system with constraints: Four recipes are shown in a) together with their primary metric (CTR, short for click-through rate) and their two constraint metrics on recipe freshness and dish type (dessert or main). The two available recommendation slots are shown in b) together with their respective attention scores. Assume we seek a ranking of two of the four available recipes to maximize CTR, with an overall freshness of at least 0.2 , while ensuring diversity. The optimal ranking is highlighted in green in c). Two additional rankings illustrate infeasible solutions to the constrained ranking problem that violate diversity/freshness constraints.

\section{FORMULATION OF THE CONSTRAINED RANKING PROBLEM}

We consider a setup where a list of items is recommended to a user (for example, a list of movies/apps). Typically, a user would pay more attention to the items in the beginning of the list rather than the end [8]. Thus, it is preferable to recommend the most desirable items (for instance, the ones that the user is most likely to be interested in) at the beginning of the list. However, in most recommendation systems, there are multiple criteria to balance while making recommendations. For example, when recommending movies, the system may want to ensure diversity in the movie genres recommended to the user. Thus, it is important to consider constraints on the ranking, which ensure that the ranking satisfies desired diversity or fairness conditions. A depiction of the ranking problem is available in figure 1 . 


\subsection{Problem description}

Consider the problem of finding a ranking of $n$ items subject to $m$ constraints. The mathematical formulation of the problem involves the following components:

Space of rankings: We denote the space of rankings $\mathbb{P}_{n}$ elements of this space are denoted $r \in \mathbb{P}_{n}$ where $r$ is a vector of length $n$ such that $r_{i}$ denotes the position of the $i$-th item in the ranking. We will interchangably represent rankings as vectors of length $n$ and as $n \times n$ permutation matrices $R$ (matrices where every row and column has exactly one entry equal to 1 and zeros elsewhere). We denote the space of $n \times n$ permutation matrices by $\mathbb{P}_{n n}$.

Attention scores: To model the importance of the position at which an item is placed, we introduce a vector $a$ of attention scores where $a[i]$ is the probability that a user would pay attention to the item at position $i$ in the ranking. Without loss of generality, we will assume that the attention scores are arranged in descending order.

Primary metric: This is the objective that is sought to be optimized by the recommender system (for example, click-throughrate). The primary metric evaluated on the $n$ items is denoted by a vector $c \in \mathbb{R}^{n}$.

Constraint metrics: Consider the problem of movie recommendation: we may have groups of items (genres of movies for example) and have a requirement that each group should have some minimum level of representation in the list of recommendations - this is an inequality constraint. We may also have equality constraints that represent fairness - for example, that two groups have equal representation in the list of recommendations. We model these via $m$ constraint metrics and adopt the convention that the first $m_{\text {eq }}$ constraints are equality constraints and the remaining are inequality constraints. We denote the values of the constraint metrics evaluated at the $n$ items by a matrix $M \in \mathbb{R}^{m \times n}$. We denote by $\theta \in \mathbb{R}^{m}$ the value of the constraint threshold, the minimum value allowed for each of the constraint metrics (for inequality constraints) or the exact value required (for equality constraints). We denote by $M_{\text {eq }}$ the $m_{\text {eq }} \times n$ submatrix of $M$ corresponding to the equality constraints and by $\theta_{\text {eq }} \in \mathbb{R}^{m_{\text {eq }}}$ the corresponding constraint values.

Notation: Before we formally define the problem, we introduce some notation that will be used throughout the paper. sort $(x)$ refers to the sorted version of the vector $x \in \mathbb{R}^{n}$ (i.e. the vector with the same coordinates as $x$ but sorted in a non-ascending order). For a vector $x \in \mathbb{R}^{n}, x_{i}$ refers to its $i$-th coordinate. However, to avoid double subscripts when working with the attention scores $a$, we use $a\left[r_{i}\right]$ to denote the $r_{i}$-th entry of the vector $a$ under ranking $r$. 1 denotes a vector in $\mathbb{R}^{n}$ with all entries equal to 1 . For any positive integer $n$, we denote by $[n]$ the set $\{1,2, \ldots, n\}$ of integers up to $n$.

2.1.1 Mathematical formulation. The constrained ranking problem can be defined as follows:

$$
\begin{aligned}
\max _{r \in \mathbb{P}_{n}} & \sum_{i=1}^{n} c_{i} a\left[r_{i}\right] \\
\text { Subject to } & \sum_{i=1}^{n} M_{k i} a\left[r_{i}\right]=\theta_{k} \quad k \in\left[m_{\mathrm{eq}}\right] \\
& \sum_{i=1}^{n} M_{k i} a\left[r_{i}\right] \geq \theta_{k} \quad k \in[m] \backslash\left[m_{\mathrm{eq}}\right]
\end{aligned}
$$

This formulation has several disadvantages: 1) The complexity would grow combinatorially with the number of items (as $n !$ ) and 2) When there are two constraint metrics that are anti-correlated, i.e., items that score high on one constraint metric tend to score low on the other, it may be impossible to pick a single ranking such that both metrics score high. In order to remedy these issues, we consider a probabilistic relaxation of (2). The probabilistic formulation is easily derived from a reformulation of the problem in terms of permutation matrices:

$$
\begin{gathered}
\max _{R \in \mathbb{P}_{n n}} c^{T} R a \\
\text { Subject to } M_{\text {eq }} R a=\theta_{\text {eq }} \\
\quad M_{\text {neq }} R a \geq \theta_{\text {neq }}
\end{gathered}
$$

Its easy to see that if $R$ is the permutation matrix corresponding to ranking $r$ (i.e. we set $R_{i r_{i}}=1$ and $R_{i j}=0$ for $j \neq r_{i}$ ) (2) is simply a restatement of (1).

2.1.2 Probabilistic relaxation. Suppose that rather than picking a deterministic ranking, we were allowed to pick a distribution over rankings. Choose $\alpha_{1}, \alpha_{2} \ldots$ such that $\sum_{j=1}^{n !} \alpha_{j}=1, \alpha_{j} \geq 0$. This defines a probabilistic ranking where we choose ranking $i$ (denoted by permutation matrix $R^{j}$ ) with probability $\alpha_{j}$ (note here that each $j$ indexes a different ranking). Then, the expected value of the constraint metrics is $\sum_{j=1}^{n !} \alpha_{j} M R^{j} a=M\left(\sum_{j=1}^{n !} \alpha_{j} R^{j}\right) a$ and a similar expression holds for the objective as well. Let $P_{\alpha}=\sum_{j=1}^{n !} \alpha_{j} R^{j}$. Then, we can rewrite this as an optimization problem over $P=P_{\alpha}$ as follows:

$$
\begin{aligned}
& \max _{P} c^{T} P a \\
& \text { Subject to } M_{\mathrm{eq}} P a=\theta_{\mathrm{eq}} \\
& M_{\text {neq }} P a \geq \theta_{\text {neq }} \\
& \quad \begin{array}{l}
\exists \alpha \text { s.t } \quad \sum_{j=1}^{n !} \alpha_{\alpha}=1 \\
\end{array}
\end{aligned}
$$

The constraints (3d) can be shown to be equivalent to the following set of constraints on $P: P 1=1, P^{\top} \mathbf{1}=1, P \geq 0$ by the BirkhoffVon Neumann theorem [12]. The above constraints are equivalent to requiring that $P$ is a doubly stochastic matrix (a non-negative square matrix whose rows and columns add up to 1).

Thus, we can simply optimize over $P$ satisfying the above constraints instead of over $\alpha$, which reduces the number of variables to $n^{2}$ (rather than $n$ !). The resulting optimization problem can be written in vector form as follows:

$$
\begin{gathered}
\max _{P} c^{\top} P a \\
\text { subject to } M_{\mathrm{eq}} P a=\theta_{\mathrm{eq}} \\
M_{\text {neq }} P a \geq \theta_{\text {neq }} \\
P 1=1, P^{\top} \mathbf{1}=1, P \geq 0
\end{gathered}
$$

2.1.3 Comparison to [19]: This formulation is nearly identical to that of Singh and Joachims [19], the key difference being that the attention scores $a$ are shared among the primary and constraints expectations. While all the use cases described in [19] have this property, their approach can deal with different attention weights 
while ours depends on them being the same. The key contribution we make is that when this special structure is present, we can develop very efficient specialized solvers that enable significant speed-ups compared to passing (4) to an off-the-shelf LP solver.

\section{FAST ALGORITHM FOR CONSTRAINED RANKING}

We derive an efficient algorithm for solving (4) by exploiting the structure of the dual problem which we describe in the following subsection.

\subsection{Dual problem}

We consider the dual of (4) with respect to the constraints (4b), (4c). The Lagrangian dual given a vector of dual variables $\lambda \in \mathbb{R}^{m}$ can be written as:

$$
\begin{array}{r}
\max _{P}\left[c^{\top} P a+\lambda^{\top} M P a-\lambda^{\top} \theta\right] \\
\text { subject to } P \text { is doubly stochastic } \\
\lambda_{\text {neq }} \geq 0
\end{array}
$$

where $\lambda_{\text {neq }}$ is the subset of $\lambda$ corresponding to the inequality constraints. Throughout this section, we use the notation

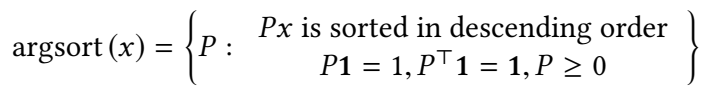

The maximization over $P$ in (5c) can be solved in closed form and corresponds to setting $P$ such that $P^{\top}\left(M^{\top} \lambda+c\right)$ and $a$ have the same order of entries, i.e, that the entries of $P^{\top}\left(M^{\top} \lambda+c\right)$ are sorted in descending order. The resulting optimum can be written as

$$
\left(\operatorname{sort}\left(M^{\top} \lambda+c\right)\right)^{\top} a-\lambda^{\top} \theta .
$$

The dual optimization problem can then be stated as:

$$
\min _{\lambda: \lambda_{\text {neq }} \geq 0} L(\lambda)=\operatorname{sort}\left(M^{\top} \lambda+c\right)^{\top} a-\lambda^{\top} \theta
$$

It follows from duality theory for convex optimization [5] that (6) and (4) have the same solution and that a solution to (4) can be recovered from a solution to (6). We note a few advantages of the dual optimization problem:

1 The number of variables in (2) is $n^{2}$ (since the variable being solved for is an $n \times n$ doubly stochastic matrix) while in (6) it is just $m$. For most recommender systems we would have $m<<n$ and hence this is a huge gain in terms of computational complexity.

2 The dual optimization problem (6) is a convex optimization problem, since the objective is a maximum over affine functions of $\lambda[5]$.

3 The objective function and its subgradients can be evaluated in $O(\max (n \log (n), m n))$ time, since it simply requires sorting a vector of length $n$ and performing two dot products.

\subsection{NodeHopper algorithm: Theoretical foundations}

The optimization problem (6) can be solved easily via off-the-shelf convex optimization methods, including reformulating it as a linear program. However, these techniques may be slow in the context of a latency constrained application (see Section 4). Further, standard methods for solving non-smooth convex optimization problems (like projected subgradient methods and their variants) are unreliable in a latency-constrained setting as their running time can depend significantly on the problem data [20].

We develop a specialized algorithm that leverages the simple structure of the optimization problem (6). We introduce some theoretical foundations that will enable us to derive the NodeHopper algorithm. We focus on the case where all the constraints are equality constraints:

$$
\begin{gathered}
\max _{P} c^{\top} P a \\
\text { subject to } M_{\mathrm{eq}} P a=\theta_{\mathrm{eq}} \\
\quad P \mathbf{1}=\mathbf{1}, P^{\top} \mathbf{1}=\mathbf{1}, P \geq 0
\end{gathered}
$$

so that the dual problem (6) is unconstrained:

$$
\min _{\lambda} L(\lambda)=\operatorname{sort}\left(M^{\top} \lambda+c\right)^{\top} a-\lambda^{\top} \theta
$$

In section 3.3.1, we describe a simple modification that allows us to deal with constrained dual optimization, albeit heuristically. We begin by introducing concepts that will be used in the NodeHopper algorithm:

Definition 3.1. Tie: A tie is a pair of item indices $(i, j)(i<j$, $i, j \in[n])$ for which the value in the ranking vector $s=c+M^{\top} \lambda$ is the same, that is $s_{i}=s_{j}$.

Definition 3.2. Irreducible ties: Given a set of ties $S=\left\{\left(i_{1}, j_{1}\right) \ldots\left(i_{k}, j_{k}\right)\right\}$ denote by $\Delta(S)$ the $n \times k$ matrix with entries

$$
[\Delta(S)]_{i, l}= \begin{cases}1 & \text { if } i=i_{l} \\ -1 & \text { if } i=j_{l} \\ 0 & \text { otherwise }\end{cases}
$$

The set of ties $S$ is called irreducible if $\Delta(S)$ has $\operatorname{rank}|S|=k$.

The idea captured in the above definition is that a set of ties is non-redudant, in that none of the ties in the set are implied by other ties (for example, the set of ties $(1,2),(2,3),(3,1)$ does not satisfy this as the third tie is implied by the first two).

Our algorithm will work under the following assumption:

Assumption 1. For every irreducible set of ties $S$ with $|S|=m$, the matrix $M \Delta(S)$ has rank $m$.

This is a non-degeneracy assumption on $M$ that can be ensured easily in practice by adding a small amount of Gaussian noise to the entries of $M$.

Definition 3.3. Node: A point $\lambda \in \mathbf{R}^{m}$ is called a node if the set $\operatorname{Ties}(\lambda)=\left\{(i, j): i<j, i, j \in\{1, \ldots, n\},\left(M_{i}-M_{j}\right)^{\top} \lambda=c_{j}-c_{i}\right\}$. satisfies that $M \Delta$ (Ties $(\lambda))$ has rank $m$. Further, if Assumption 1 is true, for any set of ties $S \subseteq \Delta$ (Ties $(\lambda)$ ) such that $|S|=m$ and $S$ is irreducible, $\lambda$ is the unique solution of the following system of linear equations:

$$
M \Delta(S) \lambda=(\Delta(S))^{\top} c
$$

The next relevant idea is that of neighboring nodes. Intuitively, neighboring nodes are the ones obtained by replacing one of the ties corresponding to a node with a different tie, inspired by the idea of a local neighborhood search in a combinatorial optimization problem. The formal definition follows: 


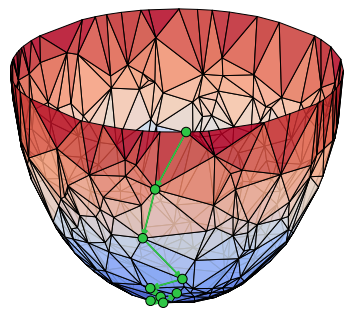

Figure 2: Cartoon depiction of the NodeHopper algorithm. Green dots show the nodes that the algorithm hops between to reach the optimal solution.

Definition 3.4. Neighboring node: Let $\lambda \in \mathbb{R}^{m}$ be a node. For every set of ties $S^{\prime}$ that satisfies $\left|S^{\prime}\right|=m$ and $S^{\prime}$ is irreducible, the unique solution $\lambda^{\prime}$ to $M \Delta\left(S^{\prime}\right) \lambda^{\prime}=\left(\Delta\left(S^{\prime}\right)\right)^{T} c$ is called a neighboring node of $\lambda$ provided that the following conditions hold:

$$
\begin{aligned}
& \exists S \subseteq \operatorname{Ties}(\lambda) \text { such that } \\
& \quad|S|=m, S \text { is irreducible, }\left|S \backslash S^{\prime}\right|=\left|S^{\prime} \backslash S\right|=1 \\
& \alpha \lambda+(1-\alpha) \lambda^{\prime} \text { is not a node for any } \alpha \in(0,1)
\end{aligned}
$$

We denote the set of neighboring nodes of $\lambda$ by $\mathcal{N}(\lambda)$.

With this definition in mind, we have the following theorem which proves that (8) can be solved by hopping between nodes, moving from a node to a neighboring node until we find a node such that all its neighbors have an equal or higher objective value, at which point we have found the optimal solution.

Theorem 3.5. Suppose (7) is feasible and Assumption 1 holds. Then, the set of nodes is finite and nonempty. Further, any algorithm that computes a sequence of values $\left\{\lambda^{k}\right\}$ such that each $\lambda^{k}$ is a node and

$$
\lambda^{k+1} \in\left\{\lambda \in \mathcal{N}\left(\lambda^{k}\right): L(\lambda)<L\left(\lambda^{k}\right)\right\}
$$

and terminates when no such update is possible, converges in a finite number of steps to an optimal solution $\lambda^{*}$ to (8).

Proof. See section 3.4.

3.2.1 Connection to conventional optimization methods. The dual problem (8) can be rephrased as a linear program:

$$
\begin{gathered}
\min _{\mu, \lambda} \mu-\theta^{\top} \lambda \\
\text { Subject to } \mu \geq\left(M^{\top} \lambda+c\right)^{\top} P a \quad \forall P \in \mathbb{P}_{n n}
\end{gathered}
$$

This is an LP with $n$ ! constraints that can be solved efficiently by a constraint generation method [13] where a subset of $m+1$ constraints is selected at each step and by enforcing those constraints to be tight, we get $m+1$ linear equations which uniquely determine $\mu, \lambda$. The constraint generation algorithm proceeds by dropping one of the constraints and replacing it with another until the optimal solution is found. The NodeHopper algorithm can be viewed as making a specific choice for how to pick this subset of constraints and decide which constraint to replace when making a move.

\subsection{NodeHopper algorithm: Practical implementation}

The pseudo-code is presented in Algorithm 1. At each iteration, the algorithm explores the set of neighboring nodes of a given node, i.e. the ones obtained by relaxing one of the ties in the node and replacing it with a different tie.

We describe the key algorithm subroutines in detail below.

JUMPTONODE. Algorithm 1 is initialized with a vector $\lambda$ which might not be a node, so we need to find a close one. We implement a fast heuristic for this purpose: sort nodes by $s=c+M^{\top} \lambda$, pick $m$ consecutive ties with the smallest gaps in $s$, such that $\left|s_{i}-s_{i+1}\right|$ is the least among all consecutive pairs. Then we solve a linear system of equations to find a node $\lambda^{*}$, as in (9).

NEIGHBORINGNODES. From Definition 3.4, all neighboring nodes contain $m-1$ irreducible ties of the current node, therefore they exist in the null-space of $M \Delta\left(\left\{\left(i_{1}, j_{1}\right), \ldots,\left(i_{m-1}, j_{m-1}\right)\right\}\right)$. Every null-space of $m-1$ irreducible ties is 1-dimensional and contains $O\left(n^{2}\right)$ nodes, but we are interested only in the neighboring ones. These can be found efficiently as described in Appendix A.

PrimalSolution. When we have a solution to the dual $\lambda^{\star}$, we know that primal solution $P^{\star} \in \operatorname{argsort}\left(M^{\top} \lambda^{\star}+c\right)$, but we still have to recover it. In order to do that, we reduce the original LP (4a) to a smaller one, given that we know values of $P^{\star}$ for non-tied entries. We describe the details in Appendix B.

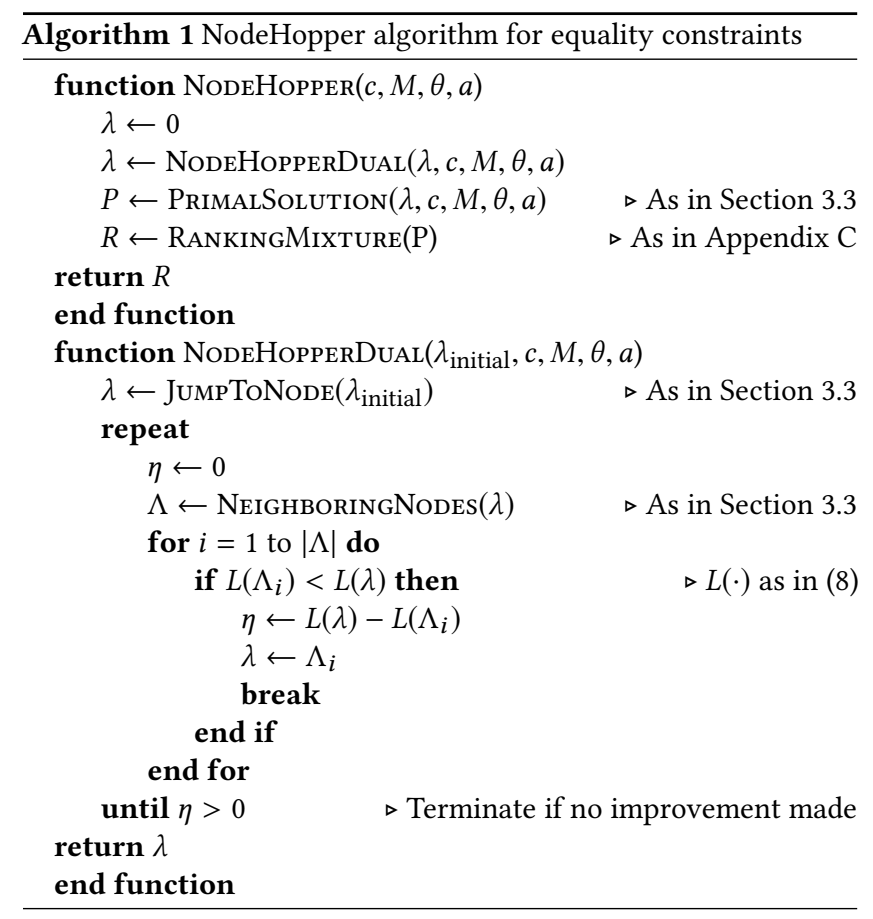

3.3.1 Constraints in the dual. If the original dual problem (6) has some constrained $\lambda$, we can still apply algorithm 1 as follows. At the optimal solution, some set of constraints in (4) would be active or binding (i.e. satisfied with exact equality). Thus, these constraints 
can be replaced with equality constraints and the remaining constraints can be dropped (since they do not affect the optimal solution). Thus, we obtain a modified problem with only equality constraints. Of course, in general, the set of active constraints is unknown and hard to guess. Thus, we use the following heuristic: we start by assuming that all the constraints are active and run algorithm 1 . If the algorithm terminates with some negative $\lambda$, we infer that the corresponding constraints were inactive, drop those and restart the algorithm. We terminate when the algorithm has found a solution with all $\lambda$ 's non-negative; the resulting algorithm is presented in Algorithm 2 in the Appendix.

3.3.2 How to detect infeasibility. The dual optimization problem (6) is bounded if and only if (4) is feasible [5]. Even with unbounded problem if Assumption 1 holds, we have a finite number of nodes and the algorithm terminates. After it terminates we check the feasibility of the primal solution $P$ w.r.t. constraints in (4). If it is not feasible we can guarantee that the problem (4) is infeasible if only equality constraints are present. For the case with inequality constraint we employ a heuristic to find a subset of the active constraints; in this case we cannot guarantee that the problem (4) is infeasible, but in practice our heuristic works in more than $99.9 \%$ of the problems we tested on, see Section 4.

3.3.3 Heuristic runtime improvement techniques. It is sufficient to consider all neighboring nodes in Algorithm 1, but for practical purposes it is beneficial to consider other additional nodes as well In the NeIGHBORINGNoDEs subroutine we find $n$ nodes along a direction; besides checking neighbouring nodes for a reduction in the objective, we can also check nodes which are further away. We can jump directly to the node which reduces the objective the most, and thus accelerate the convergence.

Another heuristic speed-up technique is to change the order in which we investigate potential neighboring nodes direction. We found that ordering them proportionally to the cosine similarity to the negative subgradient (which can be efficiently computed in $O(\max (n \log (n), m n)))$, brings significant reduction in number of iterations required for NodeHopper to converge to the global optimum.

3.3.4 Per-iteration complexity analysis. At every call to NeIGHBorINGNODES we find the null-space of a $m-1 \times m$ matrix which is $O\left(m^{3}\right)$ for every direction ( $m$ directions for nodes with disjoint ties and $2^{m}$ directions for nodes with $m$ consecutive ties) and compute values of the dual (6) at a node which is $O(\max (n \log n, n m))$. This makes the overall iteration complexity: $O\left(\max (n \log n, n m) m^{3} 2^{m}\right)$. Our experiments in Section 4 show that we seldom encounter the need to enumerate $2^{m}$ directions suggesting that the practical complexity scales better than the worst case bound.

\subsection{Proof of theorem 3.5}

Proof that set of nodes is finite and nonempty: The number of nodes is clearly finite, since each node can be uniquely determined by the set of irreducible ties of cardinality $m$ and there are at most $\left(\begin{array}{l}K \\ m\end{array}\right)$ (where $K=\left(\begin{array}{l}n \\ 2\end{array}\right)$ ) such sets. Further, by Assumption 1, each irreducible set of cardinality $m$ corresponds to a node. So, the set of nodes is non-empty and finite.

Proof that algorithm terminates: Since the number of nodes is finite and the algorithm always seeks a neighboring node with lower objective value, the algorithm must terminate in a finite number of steps. Further, when it terminates, the solution $\lambda^{\star}$ found must satisfy

$$
L\left(\lambda^{\prime}\right) \geq L\left(\lambda^{\star}\right) \quad \forall \lambda^{\prime} \in \mathcal{N}(\lambda)
$$

Proof that the solution $\lambda^{\star}$ is optimal: Our main strategy is to prove that the directional derivatives of $L(\lambda)$ at $\lambda^{\star}$ along any direction $d$ are non-negative, showing that there is no local descent direction at $\lambda^{\star}$. Since the objective is convex, this guarantees that $\lambda^{\star}$ is also globally optimal. This is a more involved proof, so we break it down for ease of readability.

Characterizing directional derivatives along neighborhood moves: We know from (12) that the neighboring nodes to $\lambda^{\star}$ have an equal or higher objective value. We use this information to characterize the directional derivative along the direction going from $\lambda^{\star}$ to each of its neighbors. To simplify calculations, we will assume that Ties $\left(\lambda^{\star}\right)$ is irreducible and consists exactly of $m$ pairs of disjoint ties (the proof for the more general case is similar but the calculations are more involved). We then prove that these directional derivatives can be used to bound the directional derivative along an arbitrary direction.

Let $I^{\star}=\left\{\left(i_{1}, j_{1}\right),\left(i_{2}, j_{2}\right), \ldots,\left(i_{m}, j_{m}\right)\right\}=$ Ties $\left(\lambda^{\star}\right)$. Let $\lambda^{\prime} \in$ $\mathcal{N}\left(\lambda^{\star}\right)$ and suppose that the $\lambda^{\prime}$ was obtained by replacing $\left(i_{k}, j_{k}\right)$ with a different tie. Let

$$
I^{k}=I^{\star} \backslash\left\{\left(i_{k}, j_{k}\right)\right\}
$$

Then, from the definition of $\mathcal{N}(\lambda), \lambda^{\prime}-\lambda^{\star}$ lies in the nullspace of the matrix $M \Delta\left(I^{k}\right)$. Denote a unit-vector in the 1-dimensional of $M \Delta\left(I^{k}\right)$ by $e_{k}$. Then, we have that $e_{1}, \ldots, e_{m}$ constitute a basis of $\mathbb{R}^{m}$ (by assumption 1).

The neighboring nodes are located along one of the following search directions in the set

$$
S=\left\{e_{1},-e_{1}, e_{2},-e_{2}, \ldots, e_{m},-e_{m}\right\}
$$

Since there are no nodes on the line segment between two neighbors, the objective function is linear on this line segment. Hence, the directional derivative has the same sign as the change in the objective value, which allows us to conclude that

$$
L^{\prime}\left(\lambda^{\star} ; d\right) \geq 0 \quad \forall d \in S
$$

Characterizing directional derivatives along an arbitrary direction: For any convex function $f$, the direction derivative at $x$ along a direction $d$ is given by the formula [20]: $\sup _{y \in \partial f(x)} d^{\top} y$ where $\partial f(x)$ is the subdifferntial of $f$ at $x$, or the set of all subgradients of $f$ at $x$. Applying this formula to $L$, we obtain

$$
L^{\prime}\left(\lambda^{\star} ; d\right)=\sup _{P \in \operatorname{argsort}\left(M^{\top} \lambda^{\star}+c\right)} d^{T}(M P a-\theta)
$$

Since $I^{\star}$ consists of disjoint ties, it can be shown that

$$
\begin{aligned}
& \operatorname{argsort}\left(M^{\top} \lambda^{\star}+c\right)= \\
& \left\{\sum_{k}\left(\left(\begin{array}{cc}
p_{k} & 1-p_{k} \\
1-p_{k} & p_{k}
\end{array}\right)\right)_{\left(i_{k}, j_{k}\right)}+B: p_{1}, \ldots, p_{m} \in[0,1]\right\}
\end{aligned}
$$


where $B$ is a fixed $n \times n$ matrix (whose exact value does not matter for this proof) and

$$
\left(\left(\begin{array}{ll}
\beta_{11} & \beta_{12} \\
\beta_{21} & \beta_{22}
\end{array}\right)\right)_{(i, j)}
$$

denotes an $n \times n$ matrix (say $J$, for brevity) such that $J$ has all entries equal to 0 except the following four non-zero entries:

$$
J_{i i}=\beta_{11}, J_{i j}=\beta_{12}, J_{j i}=\beta_{21}, J_{j j}=\beta_{22}
$$

Based on this, we can rewrite the directional derivative expression (14) as:

$$
\begin{array}{r}
\sup _{p_{1}, \ldots, p_{m} \in[0,1]} d^{T}\left(\sum_{k=1}^{m} M_{i_{k}} a_{j_{k}}+M_{j_{k}} a_{i_{k}}+M B a-\theta\right) \\
+\sum_{k=1}^{m} d^{T}\left(M_{i_{k}}-M_{j_{k}}\right)\left(a_{i_{k}}-a_{j_{k}}\right) p_{k} \\
=d^{T}\left(\sum_{k=1}^{m} M_{i_{k}} a_{j_{k}}+M_{j_{k}} a_{i_{k}}+M B a-\theta\right) \\
+\sum_{k=1}^{m} \max \left(d^{T}\left(M_{i_{k}}-M_{j_{k}}\right)\left(a_{i_{k}}-a_{j_{k}}\right), 0\right)
\end{array}
$$

Proving that all directional derivatives are non-negative:

Since $\left\{e_{1}, e_{2}, \ldots, e_{m}\right\}$ is a basis of $\mathbb{R}^{m}$, every direction $d$ can be written in the form $\sum_{k=1}^{m} \beta_{k} e_{k}$. Expressed in terms of $\beta_{k}$, the expression for the directional derivative takes the form:

$$
\sum_{k} \beta_{k} \mu_{k}+\max \left(\beta_{k} v_{k}, 0\right)
$$

where

$$
\begin{aligned}
\mu_{k} & =e_{k}^{T}\left(\sum_{k=1}^{m} M_{i_{k}} a_{j_{k}}+M_{j_{k}} a_{i_{k}}+M B a-\theta\right) \\
v_{k} & =e_{k}^{T}\left(M_{i_{k}}-M_{j_{k}}\right)\left(a_{i_{k}}-a_{j_{k}}\right)
\end{aligned}
$$

where the expression for $v_{k}$ follows from the fact that each $e_{j}(j \neq k)$ is orthogonal to $M_{i_{k}}-M_{j_{k}}$ as it lies in the subspace of $M \Delta\left(I^{j}\right)$, one of whose rows is precisely $M_{i_{k}}-M_{j_{k}}$. Plugging in $e_{k},-e_{k}$ into (15), it can easily be seen that

$$
\begin{aligned}
& L^{\prime}\left(\lambda^{\star} ; e_{k}\right)=\mu_{k}+\max \left(v_{k}, 0\right) \\
& L^{\prime}\left(\lambda^{\star} ;-e_{k}\right)=-\mu_{k}+\max \left(-v_{k}, 0\right) .
\end{aligned}
$$

Thus, we have that

$$
\beta_{k} \mu_{k}+\max \left(\beta_{k} v_{k}, 0\right)=\left\{\begin{array}{l}
\left|\beta_{k}\right| L^{\prime}\left(\lambda^{\star} ;-e_{k}\right) \text { if } \beta_{k} \leq 0 \\
\left|\beta_{k}\right| L^{\prime}\left(\lambda^{\star} ; e_{k}\right) \text { if } \beta_{k}>0
\end{array}\right.
$$

Thus, using (13), we have for every $k$ that

$$
\beta_{k} \mu_{k}+\max \left(\beta_{k} v_{k}, 0\right) \geq 0
$$

Thus,

$$
L^{\prime}\left(\lambda^{\star}, d\right)=\sum_{k} \beta_{k} \mu_{k}+\max \left(\beta_{k} v_{k}, 0\right) \geq 0
$$

for every direction $d \in \mathbb{R}^{m}$. Hence, $\lambda^{\star}$ is the optimal solution to (8). A similar argument goes through even if there are consecutive ties, with the structure of the argsort $M^{\top} \lambda^{\star}+c$ being slightly more cumbersome.

\section{EXPERIMENTAL EVALUATION}

We perform two kinds of experiments:

Algorithm behavior on synthetic data: We consider synthetically generated data for the metrics and attention scores and study the impact of various parameters of the synthetic generation process on the time taken to solve the problem optimally. In particular, we study the impact of rapidly vs. slowly decaying attention scores, the tightness of the constraints imposed (i.e. how difficult it is to satisfy the constraints simultaneously) and the number of items and constraints $(n, m)$. We report results on the impact of these on our solver (the NodeHopper) compared to state-of-the-art off-the-shelf LP solvers.

Run-time comparisons on real data: For real datasets, we study the quantity of interest in this paper: what fraction of the instances can a solver handle within a specified time limit.

Find more experiment details in Appendix D.

\subsection{Description of Datasets}

Synthetic: We generate synthetic problems drawing values of $c, M$ from the uniform distribution defined between 0 and 1 .

Unless specified otherwise we use discounted cumulative gain (DCG) based attention weighting $a[i]=\frac{1}{\log _{2}(i+1)}$. The thresholds of every problem are defined in the following way: let $P^{\prime}$ be a ranking based on the primary objective, then $\theta_{\text {base }}=M^{T} P^{\prime} a$ will be the values of secondary metrics with which the LP will be trivially solvable. Then the $r$-relative threshold is defined as $\theta_{r}=(1+r) \theta_{\text {base }}$ where $r \in \mathbf{R}_{+}$.

Google Play: We take a problem of ranking apps in a Google Play store given the primary objective and several secondary objective constraints specified by product requirements. We use attention weights computed from the logged data and $r$-relative thresholds as defined above.

Movielens 20M: To benchmark the performance on fairness task we simulate a collaborative filtering model via the singular value decomposition of the user-item matrix [9]. Then we enforce group fairness proposed in [19]: the main objective is the user-product similarity scores and the constraints matrix $M$ is constructed to enforce parity between exposure of various movie genres. Given a list of genres $g_{1}, g_{2}, \ldots$ we define:

$$
M_{i, k}=\left\{\begin{array}{l}
1 \text { if } \text { movie }_{k} \text { has genre } g_{i} \\
-1 \text { if } \text { movie }_{k} \text { has genre } g_{i+1}
\end{array}\right.
$$

with all other entries of $M$ equal to 0 and $\theta=0$. Then (4) with equality constraints we achieve exposure parity for the movie genres selected.

\subsection{Impact of problem data on algorithms}

We used synthetically generated problems to study the impact of various problem parameters on the performance of the three solvers (NodeHopper, Glop and Gurobi).

Impact of number of items $n$ : In Figure 3 we plot the runtime of all the algorithms as a function of the number of items $(n)$ for a fixed number of constraints $(m=5)$. This result shows that the NodeHopper is significantly faster than the general solvers, enabling real-time constrained re-ranking of up to 300 items. 


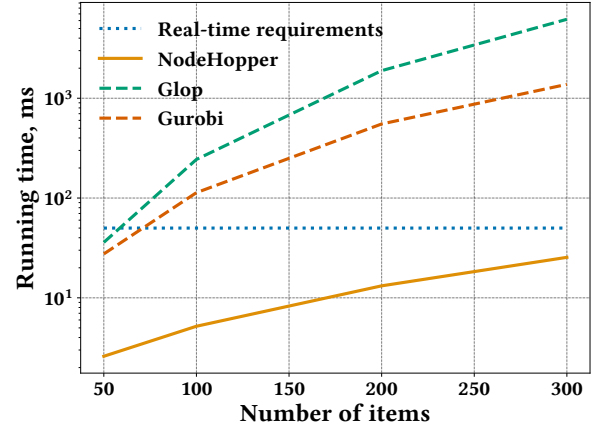

Figure 3: Number of items $(n)$ vs. running time on a synthetic dataset with five constraints $(m=5)$ and a 0.05 -relative threshold.

Impact of the relative threshold $r$ : In Figure 4 we observe running time peaks for relative thresholds around $0.05-0.10$; this is the range of $r$ where the constrained ranking problems transition to becoming infeasible, resulting in challenging problems where the solvers have to "work hard" to find feasible solutions or to prove infeasibility. Beyond this, detecting infeasibility appears easy.

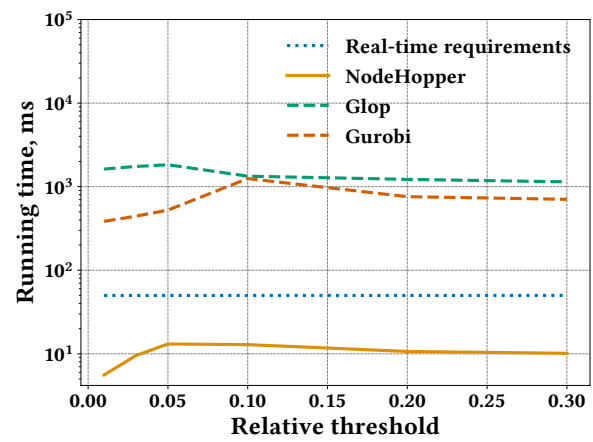

Figure 4: Relative threshold $(r)$ vs. runtime on a synthetic dataset $(n=200, m=5)$.

Impact of the attention profile: We study this by setting attention as an exponential decay $a[i]_{t}=e^{-i / t}$ which is decaying faster with small $t$ and becomes closer to linear with larger $t$. Figure 5 shows that very small values of $t$ make the problem harder for NodeHopper and simpler for Gurobi; this corresponds to a situation where the attention scores decay rapidly. However, through the range of values of $t$, the NodeHopper is consistently at least one order of magnitude faster (and often two) than Gurobi and Glop.

Feasible region of solvability within latency constraints: In Figure 6, we shade the region over $n$ and $m$ where the NodeHopper can find an optimal solution within $50 \mathrm{~ms}$. Note that the general solvers are not able to meet this constraint for any choice of $n$ and $m$.

\subsection{Performance on real datasets}

We now focus on our primary motivating question: is a given solver suitable for practical use? I.e., can it consistently solve realistic constrained ranking problems within the real-time latency constraint

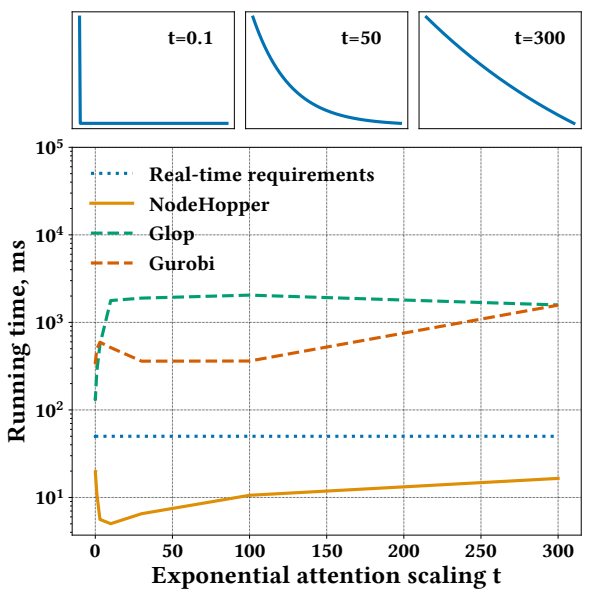

Figure 5: Runtime as a function of $t$, the parameter of an exponentially decaying attention model $a[i]=e^{-i / t}$ for a synthetic dataset $(n=200, m=5)$; attention profiles for three $t$ choices are also shown.

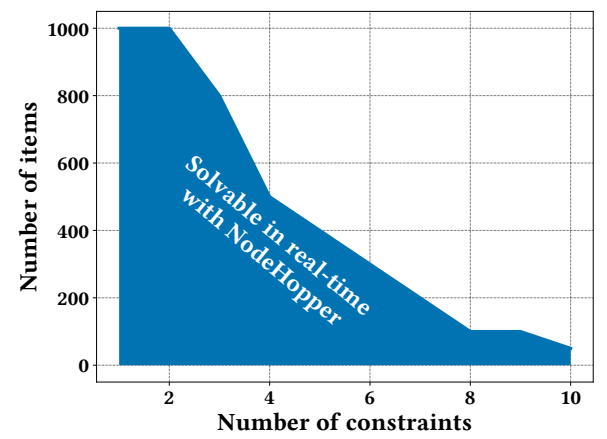

Figure 6: Shaded region over parameters $(n, m)$ of constrained ranking problems consistently solvable by the NodeHopper within the real-time constraint of $50 \mathrm{~ms}$.

of $50 \mathrm{~ms}$ ? Our experiments on real datasets show that this is indeed the case for NodeHopper, while Glop and Gurobi fail to solve the majority of such problems within the time limit.

On the Google Play dataset, we plot the fraction of instances solved within a given time limit as a function of the runtime for all three solvers in Figure 7. The results show that all three solvers are eventually able to solve more than $99.9 \%$ of the problems, but only the NodeHopper is able to consistently solve them within the time limit (and does so for $99.9 \%$ of the problems). For the MovieLens dataset, with results shown in Figure 8, the speedup of NodeHopper relative to Gurobi/Glop is even higher.

\section{CONCLUSION}

We have presented a solver that enables deploying constrained ranking under strict latency requirements. It exploits the structure of the dual optimization problem and efficiently computes a probabilistic ranking that optimizes a primary objective while satisfying several constraints (in expectation). Experiments demonstrate that our approach is the only feasible way to solve constrained ranking 


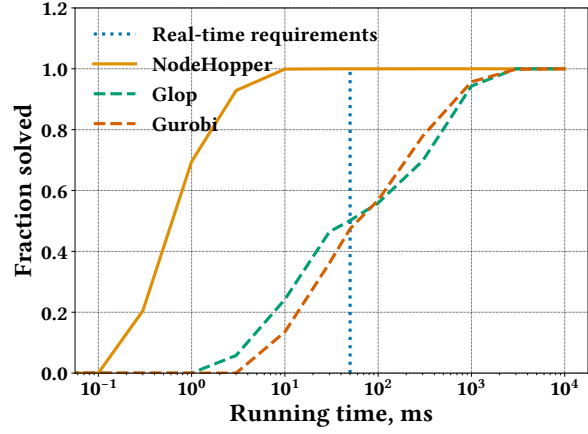

Figure 7: Fraction of problems optimally solved on the Google Play dataset with $n=200, m=1-3$.

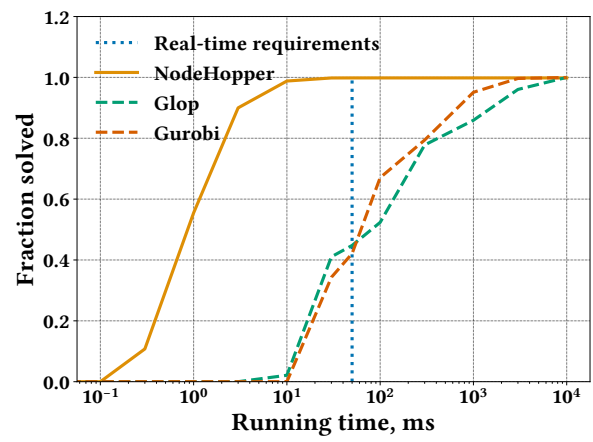

Figure 8: Fraction of problems optimally solved on the MovieLens 20M dataset with $n=50-300, m=1-4$.

problems (with constraints on diversity, fairness, freshness, etc.) within the strict latency requirements of practical systems serving live traffic. In future work, it would be interesting to generalize our solver to deal with other forms of constraints on rankings and incorporate it into learning-to-rank approaches, thereby ensuring that the learning approach produces rankings that play well with the constrained ranking post-processing step.

\section{REFERENCES}

[1] Deepak Agarwal, Shaunak Chatterjee, Yang Yang, and Liang Zhang. 2015. Constrained Optimization for Homepage Relevance. In Proceedings of the 24th International Conference on World Wide Web (Florence, Italy) (WWW 2015 Companion). Association for Computing Machinery, New York, NY, USA, 375 - 384. https://doi.org/10.1145/2740908.2745398

[2] Deepak Agarwal, Bee-Chung Chen, Pradheep Elango, and Xuanhui Wang. 2011 Click Shaping to Optimize Multiple Objectives. In Proceedings of the 17th ACM SIGKDD International Conference on Knowledge Discovery and Data Mining (San Diego, California, USA) (KDD 2011). Association for Computing Machinery, New York, NY, USA, 132-140. https://doi.org/10.1145/2020408.2020435

[3] Erling D. Anderson, Jacek Gondzio, Csaba Mészáros, and Xiaojie Xu. 1996. Im plementation of Interior-Point Methods for Large Scale Linear Programs. Springer US, Boston, MA, 189-252. https://doi.org/10.1007/978-1-4613-3449-1_6

[4] Asia J. Biega, Krishna P. Gummadi, and Gerhard Weikum. 2018. Equity of Attention: Amortizing Individual Fairness in Rankings. In The 41st International ACM SIGIR Conference on Research \& Development in Information Retrieval (Ann Arbor, MI, USA) (SIGIR '18). Association for Computing Machinery, New York, NY, USA, 405-414. https://doi.org/10.1145/3209978.3210063

[5] Stephen Boyd and Lieven Vandenberghe. 2004. Convex Optimization. Cambridge University Press, USA
[6] Chris Burges, Tal Shaked, Erin Renshaw, Ari Lazier, Matt Deeds, Nicole Hamilton, and Greg Hullender. 2005. Learning to Rank Using Gradient Descent. In Proceedings of the 22nd International Conference on Machine Learning (Bonn, Germany) (ICML '05). Association for Computing Machinery, New York, NY, USA, 89-âĂŞ96. https://doi.org/10.1145/1102351.1102363

[7] L. Elisa Celis, Damian Straszak, and Nisheeth K. Vishnoi. 2018. Ranking with Fairness Constraints. In 45th International Colloquium on Automata, Languages, and Programming (ICALP 2018) (Leibniz International Proceedings in Informatics (LIPIcs), Vol. 107), Ioannis Chatzigiannakis, Christos Kaklamanis, Dániel Marx, and Donald Sannella (Eds.). Schloss Dagstuhl-Leibniz-Zentrum fuer Informatik, Dagstuhl, Germany, 28:1-28:15. https://doi.org/10.4230/LIPIcs.ICALP.2018.28

[8] Nick Craswell, Onno Zoeter, Michael Taylor, and Bill Ramsey. 2008. An Experimental Comparison of Click Position-Bias Models. In Proceedings of the 2008 International Conference on Web Search and Data Mining (Palo Alto, California, USA) (WSDM 2008). Association for Computing Machinery, New York, NY, USA, 87-94. https://doi.org/10.1145/1341531.1341545

[9] Scott Deerwester, Susan T Dumais, George W Furnas, Thomas K Landauer, and Richard Harshman. 1990. Indexing by latent semantic analysis. Fournal of the American society for information science 41, 6 (1990), 391-407.

[10] Weicong Ding, Dinesh Govindaraj, and S V N Vishwanathan. 2019. Whole Page Optimization with Global Constraints. In Proceedings of the 25th ACM SIGKDD International Conference on Knowledge Discovery \& Data Mining (Anchorage, AK, USA) (KDD 2019). Association for Computing Machinery, New York, NY, USA, 3153-3161. https://doi.org/10.1145/3292500.3330675

[11] Iain S Duff and Jacko Koster. 2001. On algorithms for permuting large entries to the diagonal of a sparse matrix. SIAM 7. Matrix Anal. Appl. 22, 4 (2001), 973-996.

[12] Fanny Dufossé and Bora Uçar. 2016. Notes on Birkhoff-von Neumann decomposition of doubly stochastic matrices. Linear Algebra Appl. 497 (2016), 108-115.

[13] Lester Randolph Ford Jr and Delbert R Fulkerson. 1958. A suggested computation for maximal multi-commodity network flows. Management Science 5, 1 (1958), 97-101.

[14] Dietmar Jannach, Paul Resnick, Alexander Tuzhilin, and Markus Zanker. 2016. Recommender Systems - beyond Matrix Completion. Commun. ACM 59, 11 (Oct. 2016), 94-102. https://doi.org/10.1145/2891406

[15] Christopher D. Manning, Prabhakar Raghavan, and Hinrich Schütze. 2008. Introduction to Information Retrieval. Cambridge University Press, USA.

[16] Robert B. Miller. 1968. Response Time in Man-Computer Conversational Transactions. In Proceedings of the December 9-11, 1968, Fall foint Computer Conference, Part I (San Francisco, California) (AFIPS 1968 (Fall, part I)). Association for Computing Machinery, New York, NY, USA, 267-277. https: //doi.org/10.1145/1476589.1476628

[17] Filip Radlinski, Robert Kleinberg, and Thorsten Joachims. 2008. Learning Diverse Rankings with Multi-Armed Bandits. In Proceedings of the 25th International Conference on Machine Learning (Helsinki, Finland) (ICML 2008). Association for Computing Machinery, New York, NY, USA, 784-791. https://doi.org/10.1145/ 1390156.1390255

[18] Parikshit Shah, Akshay Soni, and Troy Chevalier. 2017. Online Ranking with Constraints: A Primal-Dual Algorithm and Applications to Web Traffic-Shaping. In Proceedings of the 23rd ACM SIGKDD International Conference on Knowledge Discovery and Data Mining (Halifax, NS, Canada) (KDD 2017). Association for Computing Machinery, New York, NY, USA, 405-414. https://doi.org/10.1145/ 3097983.3098025

[19] Ashudeep Singh and Thorsten Joachims. 2018. Fairness of Exposure in Rankings. In Proceedings of the 24th ACM SIGKDD International Conference on Knowledge Discovery \& Data Mining (London, United Kingdom) (KDD 2018). Association for Computing Machinery, New York, NY, USA, 2219-2228. https://doi.org/10.1145/ 3219819.3220088

[20] Jaehyun Park Stephen Boyd. 2014. Lecture note on subgradient methods. Stanford university. https://web.stanford.edu/class/ee364b/lectures/subgrad_method_ notes.pdf

[21] Masrour Zoghi, Tomas Tunys, Mohammad Ghavamzadeh, Branislav Kveton, Csaba Szepesvari, and Zheng Wen. 2017. Online Learning to Rank in Stochastic Click Models. In Proceedings of the 34th International Conference on Machine Learning - Volume 70 (ICMLâĂŻ17). JMLR.org, Sydney, NSW, Australia, 4199âĂȘ4208. 
Table 1: The best hyperparameter settings for Glop and Gurobi, as used in all experiments.

\begin{tabular}{lll}
\hline & \multicolumn{2}{c}{ Solver } \\
\cline { 2 - 3 } Hyperparameter & Glop & Gurobi \\
\hline Do presolve? & No & No \\
Do scaling? & No & No \\
LP algorithm & Primal simplex & Barrier [3] \\
\hline
\end{tabular}

\section{Appendices}

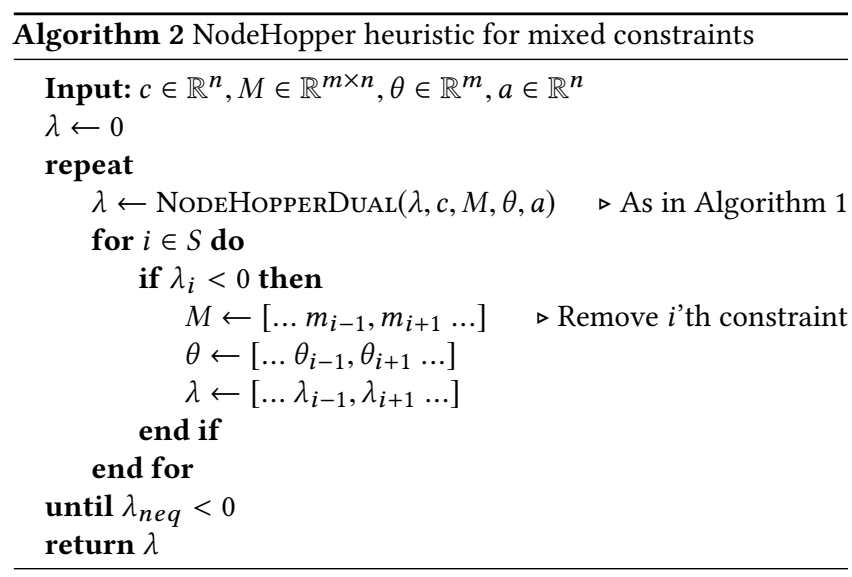

\section{A NEIGHBORINGNODES SUBROUTINE}

To find the nodes on a given direction, we compute the scoring function at a current node: $s=M^{T} \lambda+c$. The scoring is continuous, therefore the tie in the closest nodes must be one of the consecutive indices in $\operatorname{sort}(s)$. For every pair of consecutive indices $(i, j)$ we can compute the distance to the current node by evaluating:

$$
d(i, j)=\frac{s_{i}-s_{j}}{M_{i}-M_{j}} .
$$

There will be two neighboring nodes as we can always move to positive and negative directions of the line and we can compute this in $O(n m)$. Combined with finding direction, we arrive at $O\left(n m^{4} 2^{m}\right)$ to find all neighboring nodes.

\section{B PRIMALSOLUTION SUBROUTINE}

Let $G$ be a set of all item non-tied items indices and $T$ set of the tied ones. And any vector $v_{G}$ be a vector containing elements with indices in the set $G$ and matrix $A_{G}$ contain only columns with indices in the set $G$. Then $\theta_{\text {reduced }}=\theta-M_{G} P_{G}^{\star} a$ and the following LP with $m^{2}$ variables will solve for the tied part of $P^{\star}$ :

$$
\begin{gathered}
\max _{P} c_{T}^{\top} P a_{T} \\
\text { subject to } M_{T} P a_{T}=\theta_{\text {reduced }} \\
\quad P \mathbf{1}=\mathbf{1}, P^{\top} \mathbf{1}=\mathbf{1}, P \geq 0 .
\end{gathered}
$$

\section{RANKINGMIXTURE SUBROUTINE}

The result of the PrimalSolution subroutine is a doubly stochastic matrix $P$ which has $n-m$ rows with determined ranking (a value one at one of the columns) and $m$ rows and columns with stochastic rankings found. We implement a greedy heuristic [11] to do Birkhoff-von Neumann decomposition of the $m \times m$ matrix (the stochastic part) which is $O\left(\mathrm{~m}^{2}\right)$ and append fixed rankings to obtain the final distribution with $n$ items. After the decomposition we end up with at most $m^{2}$ distinct rankings. By sampling from this distribution, we can guarantee that the main objective will be maximized while the constraints are satisfied asymptotically.

\section{EXPERIMENTS DETAILS}

For fair comparison, we use a $\mathrm{C}++$ implementation of our solver and compare the running time with state-of-the-art LP solvers Glop ${ }^{1}$ and Gurobi $^{2}$ accessed via an efficient $\mathrm{C}++$ interface. Furthermore, we run all timing experiments on uniform hardware. ${ }^{3}$ We tune the hyperparameters of each solver on 100 synthetic problems with 200 items and 5 constraints with the following hyperparameters: whether to do presolve, whether to do constraints coefficients scaling, how to solve the problem - in primal, in dual or using the Barrier algorithm [3] (Gurobi only). We use the best set of the hyperparameters for all our experiments, and report them for each solver in Table 1 in the Appendix. We limit the LP solvers at maximum 10 seconds per problem and NodeHopper at 10000 iterations to cap the physical amount of time required to run experiments. NodeHopper does not have other hyperparameters, so we report the vanilla version results. All reported running times strictly include only the time spent on solving the optimisation problem, and exclude any time spent on problem data generation, reading and pre-preprocessing.

\footnotetext{
${ }^{1}$ Google's open-source linear programming system, see https://developers.google.com/ optimization/lp/glop

${ }^{2}$ Commercial solver, see https://www.gurobi.com/products/gurobi-optimizer

${ }^{3}$ All experiments were timed on the same physical machine (Intel Xeon E5-2699 CPU, 128GB RAM).
} 